\title{
PITAIA (H. costaricensis): UM FRUTO COM CARACTERÍSTICAS ATRATIVAS PARA A INDÚSTRIA DE PROCESSAMENTO
}

\author{
N. L. CRISTOFOLI ${ }^{1}$, C. A. R. LIMA ${ }^{1}$, A. M. MOTA ${ }^{1}$, N. M. PEIXOTO ${ }^{1}$, J. da S. S. LIMA ${ }^{2}$, \\ F. M. R. SILVA ${ }^{2}$, L. B. de T. VASCONCELOS ${ }^{3}$, R. W. de FIGUEIREDO ${ }^{3}$ \\ ${ }^{1}$ Graduando pela Universidade Federal do Ceará, Departamento de Tecnologia de Alimentos \\ ${ }^{2}$ Mestranda em Ciência e Tecnologia de Alimentos, Universidade Federal do Ceará, \\ Departamento de Tecnologia de Alimentos \\ ${ }^{3}$ Professor Dr. na Universidade Federal do Ceará, Departamento de Tecnologia de Alimentos \\ E-mail: nathana.c@ hotmail.com
}

\begin{abstract}
RESUMO - O mercado de frutas exóticas tem aumentado consideravelmente. A pitaia tem chamado mais atenção atualmente devido a suas características sensoriais e ao seu aspecto exótico. Neste trabalho foram realizadas as análises de sólidos solúveis, $\mathrm{pH}$, acidez total titulável, açúcares totais e redutores, vitamina $\mathrm{C}$, rendimento e firmeza em frutos de pitaia da espécie $H$. costaricensis. A pitaia é um fruto pouco ácido com valor médio de $\mathrm{pH}$ de 4,63. O valor de vitamina $\mathrm{C}$, em $100 \mathrm{~g}$, supre $60 \%$ do índice de ingestão diária (IDR), determinado segundo a legislação brasileira vigente $(45 \mathrm{mg})$. Este fruto apresenta características satisfatórias para industrialização, apresentando $83 \%$ no rendimento de polpa em frutos maduros.
\end{abstract}

\section{INTRODUÇÃO}

As características físicas e físico-químicas dos frutos são de grande importância para a sua comercialização e manuseio. A aparência externa dos frutos, tais como tamanho, consistência, espessura, forma e coloração da casca são fatores importantes para a aceitabilidade pelos consumidores (COSTA et al., 2004)

No Brasil, diferentes variedades frutíferas nativas ou exóticas, muitas vezes ainda pouco conhecidas, vêm sendo utilizadas pelas populações locais, em decorrência do grande potencial para exploração no mercado de consumo in natura e/ou para industrialização. No entanto, são necessárias ações que favoreçam o cultivo e a preservação dessas espécies, além de estudos diferenciados para determinação do potencial desses frutos, visando sua utilização no mercado de alimentos funcionais. Atualmente, observa-se uma maior demanda por alimentos funcionais, que além de complementar a dieta diária, propicia benefícios à saúde (LORENZI et al.,2006; SILVA et al., 2008).

A pitaia é um fruto exótico conhecida mundialmente como "Fruta-do-Dragão" pertencente à família Cactaceae. De acordo com a espécie, seus frutos podem apresentar características diversificadas, como formato, presença de espinhos, cor da casca e da polpa, 
refletindo em alta variabilidade genética (JUNQUEIRA et. al., 2007). São encontradas várias espécies, agrupadas em quatro gêneros principais: Stenocereus (Britton\& Rose), Cereus (Mill), Selenicereus (Riccob) e Hylocereus (Britton\& Rose) (MIZRAHI, et al., 1997; BRITTON e ROSE, 1963), destacando-se a Hylocereuscostaricensis (pitaia vermelha de polpa vermelha) e a Hylocereusundatus (pitaia vermelha de polpa branca).

Há algumas décadas, a pitaia era desconhecida e atualmente ocupa um crescente nicho no mercado de frutas exóticas da Europa e vêm sendo consumida, não só pelo exotismo da aparência, como também por suas características como sabor doce e suave, rico potencial vitamínico, polpa firme sendo constituída entre $60-80 \%$ do fruto, podendo ser apreciada na forma in natura, de sucos, geleias, doces, sorvetes, dentre outros (LE BELLEC et al., 2006; MARQUES et al., 2011; MOREIRA et al., 2011, DAM, 2009).

Informações a respeito das características físicas, físico-químicas de frutos nativos, como é o caso da pitaia, são ferramentas básicas para avaliação do consumo e formulação de novos produtos que venham atender ao mercado consumidor. No entanto, observa-se uma escassez de pesquisas relativas ao estudo deste fruto, bem como seu processamento tecnológico, o que implica no não conhecimento das potencialidades do mesmo. Portanto, a realização da presente pesquisa teve como objetivo determinar as características físicas e físico-químicas da pitaia.

\section{MATERIAL E MÉTODOS}

Foram utilizados os frutos da pitaia (H. costaricensis) adquiridas no comércio varejista de Fortaleza e encaminhadas ao Laboratório de Frutos Tropicais e Hortaliças do Departamento de Engenharia de Alimentos da Universidade Federal do Ceará. Os frutos foram lavados e sanitizados por imersão em solução de hipoclorito de sódio (200 ppm) durante 5 minutos, em seguida foram lavados em água corrente, drenados e despolpados, seguido de homogeneização da polpa em processador doméstico.

As análises físicas e físico-químicas foram realizadas na polpa dos frutos. As amostras foram submetidas a avaliações físicas de diâmetro longitudinal e transversal, a análise de cor, no qual foi realizada utilizando Colorímetro Digital MINOLTA (Osaka, Japão), as leituras foram expressas no módulo $\mathrm{L}^{*}, \mathrm{a}^{*}$ e $\mathrm{b} *$ de acordo com CIE (1986). O espaço colorimétrico CIELAB é definido pelas coordenadas $\mathrm{L}^{*}, \mathrm{a}^{*}, \mathrm{~b}^{*}$. A coordenada $\mathrm{L}^{*}$ corresponde à luminosidade, e $\mathrm{a}^{*}$ e $\mathrm{b}^{*}$ referem-se às coordenadas de cromaticidade verde (-) / vermelho (+) e azul (-) / amarelo (+), respectivamente. As análises físico-químicas constaram de determinação do pH em pHmetro Jenway 3505 pH Meter (AOAC, 1995), sólidos solúveis totais em refratômetro digital Pocket PAL-1 ATAGO (BRASIL, 2005) e acidez titulável (BRASIL, 2005), além de ácido ascórbico (ITAL, 1990) e açúcares totais e redutores (MILLER, 1959).

Todas as análises foram realizadas em triplicata. Os resultados obtidos foram expressos em média e desvio-padrão entre as amostras.

\section{RESULTADOS E DISCUSSÃO}


Estão apresentados na Tabela 1 os valores referentes às características físicas e físicoquímicas dos frutos de pitaia comercializados na cidade de Fortaleza-CE.

Tabela 1 - Características físicas físico-químicas dos frutos de pitaia comercializados na cidade de Fortaleza-CE

\begin{tabular}{|c|c|}
\hline Características Físicas & Pitaia \\
\hline Diâmetro I ${ }^{1}(\mathrm{~mm})$ & $92,64 \pm 1,6$ \\
\hline Diâmetro $\mathrm{II}^{2}(\mathrm{~mm})$ & $88,39 \pm 1,04$ \\
\hline Massa fresca $(g)^{3}$ & $403 \pm 12,66$ \\
\hline Rendimento de polpa $(\%)^{3}$ & $82,63 \pm 7,63$ \\
\hline $\mathrm{L}^{* 3}$ & $33,52 \pm 0,95$ \\
\hline$a^{* 3}$ & $3,06 \pm 0,6$ \\
\hline$b^{*^{3}}$ & $2,44 \pm 0,22$ \\
\hline $\mathrm{pH}^{3}$ & $4,63 \pm 0,03$ \\
\hline Sólidos solúveis totais $\left({ }^{\circ} \mathrm{Brix}\right)^{3}$ & $11,53 \pm 0,06$ \\
\hline Acidez total titulável - ácido cítrico $\left(\mathrm{mg} \cdot 100 \mathrm{~g}^{-1}\right)^{3}$ & $0,29 \pm 0,01$ \\
\hline Ácido ascórbico $\left(\mathrm{mg} .100 \mathrm{~g}^{-1}\right)^{3}$ & $27,07 \pm 0,41$ \\
\hline Açúcares totais $\left({\left.\mathrm{g} 100 \mathrm{~g}^{-1}\right)^{3}}^{3}\right.$ & $18,50 \pm 1,2$ \\
\hline Açúcares redutores $\left(\mathrm{g} 100 \mathrm{~g}^{-1}\right)^{3}$ & $2,41 \pm 0,18$ \\
\hline
\end{tabular}

${ }^{1}$ Longitudinal, ${ }^{2}$ Transversal ${ }^{3}$ Análise realizada na polpa do fruto

Os frutos de pitaia apresentam diâmetro médio longitudinal e transversal na ordem de $92,64 \mathrm{~mm}$ e $88,39 \mathrm{~mm}$, respectivamente. A análise dessas variáveis, isoladamente, tem pouca importância para a caracterização dos frutos, contudo, a relação entre essas variáveis é bastante representativa, pois indica o formato do fruto, e quanto mais próximo de 1, mais redondo. Nesse sentido, essa relação foi de 1,05 entre os frutos estudados. Para o rendimento da polpa, os frutos avaliados apresentaram características satisfatórias para industrialização, na forma de polpa congelada, sucos, geléias, néctares e sorvetes, devido o aspecto percentual do rendimento de polpa, em frutos maduros, na ordem de $82,63 \%$. Os valores médios de rendimento de polpa e diâmetro longitudinal foram superiores aos encontrados por Souza et. al. (2006), que obtiveram valores de 74,89\% e 83,18mm, respectivamente. As discrepâncias entre as características físicas desses frutos podem ser atribuídas às variações do tipo de solo, das condições climáticas da região de cultivo, além dos fatores genético, fisiológico e nutricional.

As coordenadas de cor obtidas para a polpa da pitaia demostram que a mesma possui luminosidade ( $\left.\mathrm{L}^{*}\right)$ da ordem de 33,52 e os valores médios de 3,06 e 2,44 para as coordenas a* e $b^{*}$, respectivamente. Os resultados obtidos evidenciam que a coloração da polpa possui tonalidade vermelha, devido $a^{*}$ possuir valor positivo, o qual supera o tom amarelo expresso pela coordenada $b^{*}$ com valor também positivo.

De acordo com os valores obtidos, os frutos de pitaia apresentaram $\mathrm{pH}$ de 4,63, semelhante ao encontrado por Rufino et. al (2007) de 4,5. Para sólidos solúveis totais os valores obtidos $\left(11,53^{\circ}\right.$ Brix $)$ estão dentro da faixa aceitável, onde frutas maduras apresentam valores médios de sólidos entre 8 e $14 \%$ estando de acordo com Chitarra\&Chitarra (2005). A acidez total titulável foi de $0,29 \mathrm{mg} .100 \mathrm{~g}^{-1}$, semelhante aos valores médios encontrado por 
Abreu et. al. (2012), de 0,20 mg.100g ${ }^{-1}$ na espécie H. undatus (conhecida como pitaia branca) a $0,24 \mathrm{mg} \cdot 100 \mathrm{~g}^{-1}$ na espécie S. polyrhizus (conhecida como pitaia vermelha).

A polpa apresentou concentração de ácido ascórbico $\left(27,07 \mathrm{mg} \cdot 100 \mathrm{~g}^{-1}\right)$ menor que os valores descritos por Choo e Yong (2011) e Mahattanatawee et al (2006) em pitaias de polpa vermelha, que encontraram teores médios iguais a $32,65 \mathrm{mg} .100 \mathrm{~g}^{-1}$ e $55,8 \mathrm{mg} \cdot 100 \mathrm{~g}^{-1}$, respectivamente.

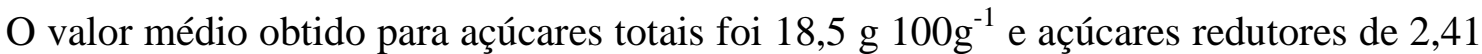
$\mathrm{g} 100 \mathrm{~g}^{-1}$. Wu et. al. (2006) encontraram valores de açúcares totais menores $\left(10,1{\left.\mathrm{~g} 100 \mathrm{~g}^{-1}\right) \mathrm{em}}^{\mathrm{m}}\right.$ pitaias vermelhas. De modo geral, os frutos tem aumento na concentração de açúcares, durante a maturação, o que na maioria das vezes ocorre, ainda, na planta, no entanto, após a colheita, principalmente, em frutos climatéricos pode acontecer síntese de açúcares, resultando na elevação dos açúcares totais (Chitarra e Chitarra, 2005). Os açúcares redutores atribuem sabor doce mais acentuado, portanto conferem aos frutos características interessantes para comercialização.

De modo geral a polpa de pitaia apresenta valor de $\mathrm{pH}$ alto $(4,63)$, baixa acidez $(0,29$ g. $\left.100 \mathrm{~g}^{-1}\right)$ comparando com outros frutos como cajá, onde Oliveira et al. (1998) encontrou valores de $\mathrm{pH}$ e acidez de 1,27 g. $100 \mathrm{~g}^{-1}$ e 2,61, respectivamente, e maracujá com valores de pH de 2,60 e acidez de 4,38 g.100 ${ }^{-1}$ (Gomes et al., 2006). Os sólidos solúveis totais na concentração de $11,53{ }^{\circ}$ Brix são devido principalmente à presença de açúcares e é considerado um valor elevado se comparado a demais frutos como a acerola $\left(6,50{ }^{\circ} \mathrm{Brix}\right.$ Oliveira et. al. 1999). Essas características tornam a polpa um meio adequado para a contaminação microbiológica, mas por outro lado, muito adequada para ser adicionado a alimentos de baixa acidez. Além disso, a baixa acidez da polpa a caracteriza como uma alternativa para a correção da acidez de outros sucos, prática comum nas indústrias de sucos de frutas.

\section{CONCLUSÃO}

Considerando os resultados encontrados, os frutos de pitaia apresentam características satisfatórias para industrialização, devido principalmente ao elevado rendimento de polpa, sendo também importante a concentração de acido ascórbico presente no fruto, na qual o consumo de $100 \mathrm{~g}$ da polpa vermelha supre $60 \%$ das recomendações diárias de vitamina $\mathrm{C}$, segundo a legislação brasileira vigente $(45 \mathrm{mg})$. Este fruto também corresponde a uma alternativa em potencial para ser utilizada nas indústrias de sucos de frutas com o objetivo de corrigir a acidez de outros sucos devido a considerável concentração de sólidos solúveis totais e a baixa acidez da polpa.

\section{REFERÊNCIAS BIBLIOGRÁFICAS}

ABREU, W.C.; LOPES, C. O.; PINTO, K. M.; OLIVEIRA, L. A.; CARVALHO, G. B. M.; BARCELO, M. F. P. Características físico-químicas e atividade antioxidante total de pitaias vermelha e branca. Rev Inst Adolfo Lutz. 2012; 71(4):656-61. 
AOAC - Association of Official Analitical Chemists - Official Methods of Analysis. Artlington, 1995.

BRASIL. Ministério da Saúde. Agência Nacional de Vigilância Sanitária. Métodos FísicoQuímicos para Análise de Alimentos / Ministério da Saúde, Agência Nacional de Vigilância Sanitária - Brasília: Ministério da Saúde, 2005. 1018p.

BRITTON N.L., ROSE J.N., Descriptions and illustrations of plants of the cactus family, Vol. I and II, Dover Publ., Inc., New York, USA, p. 183-195, 1963.

COSTA, N. P.; LUZ, T. L. B.; BRUNO, R. L. A. Caracterização físico-química de frutos de umbuzeiro (Spondias tuberosa) colhidos em quatro estádios de maturação. Bioscience Journal (Uberlândia), v. 20, n. 2, p. 65-71, 2004.

CHITARRA, M. I. F.; CHITARRA, A. B. Pós-colheita de frutos e hortaliças: fisiologia e manuseio. Lavras: ESAL/FAEPE, 2005. 785 p.

Choo WS, Yong WK. Antioxidant properties of two species of Hylocereus fruits. AdvApplSci Res. 2011;2(3):418-25.

DAM (Departament of Agriculture-Malaysia). A Research and Development Center for Pitaya (Dragon Fruit). Malásia. Disponível em: <http://www.dam- Departament of Agriculture-Malaysia/default.htm> Acesso em 15 de abr de 2009.

GOMES, T. S.; CHIBA, H. T.; SIMIONATO, E. M. R. S.; SAMPAIO, A. C. Monitoramento da qualidade da polpa de maracujá-amarelo - seleção AFRUVEC, em função do tempo de armazenamento dos frutos. Revista Alimentos e Nutrição, Bauru, v.17, n.4, p. 401-405, 2006.

ITAL - Instituto de Tecnologia de Alimentos. Manual Técnico de Analise Química de Alimentos. Campinas, 1990.

JUNQUEIRA, K. P.; FALEIRO, F, G; JUNQUEIRA, N,T,V; BELLON, G. ; FONSECA, K.G; LIMA, C.A; SANO, S.M. Diversidade genética de Pitayas nativas do cerrado com base em marcadores RAPD. In: $4^{\circ}$ CONGRESSO BRASILEIRO DE MELHORAMENTO DE

PLANTAS, 4, 2007, São Lourenço- MG. Diversidade genética de Pitayas nativas do cerrado com base em marcadores RAPD, Lavras: UFLA, 2007. CD-ROM.

LE BELLEC, F. et al. Pitahaya (Hylocereus spp.): a new crop, a market with a future. Fruits, v.61, n.4, p.237-250, 2006. Disponível em: <http://pdfcast.org/pdf/pitahaya-hylocereus-sppa-new-fruit-crop-a-market-with-a-future $>\quad$ Acesso em: 22 abril. 2014. doi: 10.1051/fruits:2006021.

LORENZI, H. et al. Frutas Brasileiras e exóticas cultivadas (de consumo in natura). São Paulo: Instituto Plantarum de Estudos da Flora, 2006.672p.

Mahattanatawee K, Manthey JÁ, Luizo G, Talcott ST, Goodner K, Balswin EA. Total antioxidante activity and fiber content of select Florida-grown tropical fruits. J Agric Food Chem. 2006;54(19):7355-63. 
MARQUES, V.B.; MOREIRA, R.A.; RAMOS, J.D; ARAÚJO, N.A.; SILVA, F.O.R. Fenologia reprodutiva de pitaia vermelha no município de Lavras, MG. Ciência Rural, Santa Maria, v.41, n.6, p.984-987, 2011.

Miller, G.L. 1959. "Use of Dinitrosalicylic Acid Reagent for Determination of Reducing Sugar." Anal. Chem. 31:426-428.

Mizrahi, Y. and A. Nerd and P.S. Nobel. 1997. Cacti as crops. Hort. Rev. 18:291-319.

MOREIRA, R.A; RAMOS, J.D.; MARQUES, V.B.; ARAÚJO, N.A.; MELO, P.C. Crescimento de pitaia vermelha com adubação orgânica e granulado bioclástico. Ciência Rural, Santa Maria, v.41, n.5, p.785-788, 2011.

OLIVEIRA, M. E. B.; BASTOS, N. S. R.; FEITOSA, T.; BRANCO, A. A. C.; SILVA, M. G, G. Avaliação de parâmetros de qualidade físico-químicos de polpas congeladas de acerola, cajá e caju. Ciênc. Tecnol. Aliment. vol.19 n.3 Campinas Sept./Dec. 1999.

OLIVEIRA, M. E. B.; FEITOSA, T.; BASTOS, M. S. R.; FREITAS, M. L.; MORAIS, A. S. Qualidade de polpas congeladas de frutas, fabricadas e comercializadas nos estados do Ceará e Rio Grande do Norte. B.CEPPA, v. 16, n. 1, Curitiba, p. 13-22, 1998.

Rufino MSM, Alves RE, Brito ES, Morais SM, Sampaio CG, Pérez-Jiménez J, et al. Metodologia científica: determinação da atividade antioxidante total em frutas pela captura do radical livre DPPH. EMBRAPA Com Técn.2007; 127:1-4.

SILVA, M. R. et al. Caracterização química de frutos nativos do cerrado.Cienc. Rural, Santa Maria, v. 38, n.6, p. 1790-1793, 2008.

SOUZA, L. S.; JUNQUEIRA, K. P.; LIMA, C. A.; CASTIGLIONI, G. L.; VILLANOVA, A. C. C.; JUNQUEIRA, N. T. V.; SILVA, D. G. P.; FALEIRO, F. G.; SANTOS, E. C.; BELLON, G.; JUNQUEIRA, L. P. Características físico-químicas de frutos de pitaya cultivada nos cerrados. In: CONGRESSO BRASILEIRO DE FRUTICULTURA, 19., 2006, Cabo Frio. Frutas do Brasil: saúde para o mundo: palestras e resumos. Cabo Frio: SBF; UENF; UFRRJ, 2006. p. 323.

Wu LC, Hsu HW, Chen YC, Chiu CC, Lin YL, Ho JÁ. Antioxidant and antiproliferative activities of red pitaya.Food Chem. 2006; 95(2):319-27. 